\title{
Characteristics and Outcomes of Patients with Acute Liver Failure Admitted to Australian and New Zealand Intensive Care Units
}

\author{
Stephen Warrillow ${ }^{1,2}$ \\ Michael Bailey ${ }^{3}$ \\ David Pilcher ${ }^{3,4}$ \\ Alex Kazemi ${ }^{5}$ \\ Colin McArthur ${ }^{6,7}$ \\ Paul Young ${ }^{7,8}$ \\ Rinaldo Bellomo $1,2,4,9,10$
}

1. Department of Intensive Care, Austin Health, Melbourne Australia

2. School of Medicine, University of Melbourne, Melbourne, Australia

3. Australian and New Zealand Intensive Care Research Centre, Monash University School of Public Health and Preventive Medicine, Melbourne Australia

4. Department of Intensive Care, Alfred Health, Melbourne Australia

5. Intensive Care Unit, Middlemore Hospital, South Auckland, New Zealand

6. Department of Critical Care Medicine, Auckland City Hospital, Auckland, New Zealand

7. Medical Research Institute of New Zealand, Auckland, New Zealand

8. Intensive Care Unit, Wellington Hospital, Wellington, New Zealand

9. Department of Intensive Care Royal Melbourne Hospital, Melbourne, Australia

10. Data Analytics Research and Evaluation (DARE) Centre, Austin Hospital and University of Melbourne, Melbourne, Australia

This is the author manuscript accepted for publication and has undergone full peer review but has not been through the copyediting, typesetting, pagination and proofreading process, which may lead to differences between this version and the Version of Record. Please cite this article as doi: $10.1111 / \mathrm{imj} .14167$

This article is protected by copyright. All rights reserved. 


\section{Corresponding author}

Stephen Warrillow

Department of Intensive Care

Austin Health

PO Box 1455

Heidelberg Victoria 3084

Australia

Email: Stephen.Warrillow@austin.org.au

Phone: +61394964728

Fax: +61394963932

Conflicts of Interest:

The authors declare that there are no relevant conflicts of interest pertaining to the content of this manuscript.

Financial Disclosure Statement:

The authors declare that there are no relevant financial disclosures to be made that are of relevance to the content of this manuscript

\section{Funding}

The Medical Research Institute of New Zealand is supported by Independent Research Organisation Funding from the Health Research Council of New Zealand. This research was conducted during the tenure of a Health Research Council of New Zealand Clinical Practitioner Research Fellowship held by PY 


\section{Abstract}

\section{Background}

Knowledge about patients with Acute Liver Failure (ALF) in Australia and New Zealand (ANZ) is lacking. We hypothesised that the pattern of disease would be similar to previous studies and that, despite low transplantation rates, mortality would be comparable.

\section{Methods}

We obtained data from the ANZ Intensive Care Society Adult Patient Database and the ANZ Liver Transplant Registry for ten years commencing 2005 and analysed for patient outcomes.

\section{Results}

During the study period, 1022698 adults were admitted to intensive care units (ICUs) across ANZ, of which 723 had ALF. The estimated annual incidence of ALF over this period was 3.4/million people and increased over time $(p=0.001)$. ALF patients had high illness severity (APACHE III 79.8 vs. 50.1 in non-ALF patients; $p<0.0001$ ), and were more likely to be younger, female, pregnant and immunosuppressed. ALF was an independent predictor of mortality (OR $1.5(1.26-1.79) ; \mathrm{p}<0.0001)$. At less than $23 \%$, the use of liver transplantation was low, but the mortality of $39 \%$ was similar to previous studies.

\section{Conclusions}

ALF is a rare but increasing diagnosis in ANZ ICUs. Low transplantation rates in ANZ for ALF do not appear to be associated with higher mortality rates than reported in the literature.

Key words: acute liver failure, paracetamol overdose, emergency liver transplantation 


\section{Introduction}

Acute liver failure (ALF) is relatively uncommon ${ }^{1}$, but leads to a characteristic pattern of critical illness with multiple organ failure and a high associated mortality ${ }^{2,3}$ in the absence of transplantation $^{4-6}$. Admission to an intensive care unit (ICU) is almost always required ${ }^{3}$. Despite the potential risk of a poor outcome and a period of extreme critical illness, patients who survive ALF may return to good health ${ }^{7}$. While some studies evaluating the patterns of presentation and incidence of ALF have been undertaken in several regions or specialised centres $^{4-6,8-29}$, little is known about the characteristics of these patients in Australia and New Zealand $^{30,31}$. However, an understanding of the characteristics, management and outcomes of these patients would help develop a binational research agenda and interventional trials aimed at improving outcomes ${ }^{32-34}$. Accordingly, we sought to describe the key characteristics, incidence and outcomes of patients with ALF admitted to Australian and New Zealand (ANZ) ICUs over a ten-year period using data from the ANZ Intensive Care Society's (ANZICS) Centre for Outcome and Resource Evaluation (CORE) Adult Patient Database (APD).

We hypothesised that patients with ALF admitted to ANZ ICUs would have similar characteristics, and survival outcomes to those reported in previous studies from other regions despite lower emergency liver transplantation (ELT) rates.

\section{Methods}

This study was approved by the Alfred Hospital Research Ethics Committee as a low risk project without need for individual patient consent.

We conducted a retrospective cross-sectional study using data extracted from the ANZICS CORE APD. The APD is a high-quality database ${ }^{35}$ that receives prospectively collected contributions from over 180 participating ICUs across ANZ, including more than $80 \%$ of 
tertiary referral units during the study period and all five liver transplant centres. De-identified individual patient data are submitted for 109 variables.

All completed adult ICU admissions that were submitted to the APD over the period 2005 to 2014 were included in the analysis, excluding re-admission episodes to ICU during an index hospital admission and palliative care patients. Patients with ALF were identified as having admission diagnosis APACHE III-J sub-code 301.01 (Acute Liver Failure), excluding those also recorded as having cirrhosis or chronic liver disease as a pre-existing chronic health condition. We obtained data regarding baseline physiological conditions (age, gender, weight, pregnancy status), comorbidities, arterial blood gases, fraction of inspired oxygen $\left(\mathrm{FiO}_{2}\right)$, respiratory rate, need for mechanical ventilation (MV), and haemodynamic characteristics within the first 24 hours of ICU admission. In addition, we obtained data on adverse events (respiratory or cardiac arrest in the 24 hours prior to ICU admission, acute renal failure occurrence during the first 24 hours of ICU stay), ICU admission source, admission unit (ICU or high dependency unit (HDU)) and admission type (emergency response admission or not). The Acute Physiology And Chronic Health Evaluation (APACHE) III and the Australian and New Zealand Risk of Death (ANZROD) ${ }^{36}$ scores were calculated to measure critical illness severity. The key patient outcomes of interest were ICU and in-hospital mortality. Data on ICU and hospital length of stay, mortality outcome and discharge destination were also collated, with in-hospital 30-day mortality calculated by excluding deaths with a hospital length of stay greater than 30 days.

To estimate emergency transplantation rates, data on ELT use during the period analysed were obtained from the Australian and New Zealand Liver Transplant Registry ${ }^{37}$, which records all liver transplant related activity in ANZ, including all instances of liver transplantation for ALF. These data were not linked to those obtained from the APD.

This article is protected by copyright. All rights reserved. 


\section{Data Analysis}

We analysed presentation type, comorbid medical conditions, patterns of pathophysiological derangement and need for ventilator support. We evaluated differences between survivors and non-survivors and compared ALF patients with all other patients admitted with non-ALF diagnoses during the ten-year period. Data are reported as numbers and percentage for binomial variables, means with standard deviation when reporting normally distributed continuous data, and medians with interquartile ranges [IQRs] when reporting non-normally distributed data. We evaluated all hospital outcomes, including mortality and discharge destination. We performed group comparisons using chi-square tests for equal proportion, student's $t$ test for parametric variables and Wilcoxon rank sum tests otherwise. To facilitate a measure of illness severity that was independent of liver failure, patient risk of death was calculated in accordance with ANZROD ${ }^{36}$, with ALF related components removed from the calculation (hepatic failure, cirrhosis, chronic liver disease).

To determine independent predictors of mortality within the ALF population, we developed multivariable models for features at both presentation to ICU (baseline demographics) and after 24 hours. To identify independent baseline differences that may exist between ALF and non-ALF patients, a multivariable logistic regression model was developed with the resulting probability then used to reflect each patient's propensity of presenting to ICU with ALF. To determine if ALF was an independent predictor of survival amongst all ICU patients, we used both logistic and Cox-proportional hazard regression models that adjusted for year, severity of illness and propensity for ALF. Change in hospital outcomes over time were determined using logistic regression, adjusting for illness severity, propensity to present with ALF and year. To ascertain if changes in outcome over time differed between ALF and non-ALF 
patients, an interaction term between ALF and year of admission was fitted with year of admission treated both as a categorical variable and as a continuous variable. All regression models were constructed in SAS Version 9.4 (SAS Institute Inc., Cary, NC, USA) using mixed hierarchical regression with patients nested within site and site treated as a random effect. Unless otherwise stated, multivariable models were developed using stepwise selection procedures $(p<0.001)$ and validated using backwards elimination procedures with logistic regression results presented as odds ratios $(95 \% \mathrm{Cl})$ while Cox-proportional hazards regression models are presented as hazard ratios $(95 \% \mathrm{Cl})$. A two-sided $\mathrm{p}$-value of 0.01 was used to indicate statistical significance.

To calculate the population prevalence of ALF, we used end-of-year population estimates for Australians and New Zealanders above age 16 from the Australian Bureau of Statistics (3101.0 Table 59) and Statistics New Zealand Infoshare (http://www.stats.govt.nz/infoshare/ViewTable.aspx), adjusting for annual APD coverage of ANZ ICU admissions of $80 \%$. This study is reported in accordance with the STROBE statement guidelines for observational epidemiologic studies ${ }^{38}$.

\section{Results}

Identification of ALF patients

A total of $1,022,698$ patients aged 17 years or older were admitted to contributing ICUs across ANZ from 2005 until 2014 inclusive. During this period, data were submitted for an average of more than $80 \%$ of eligible intensive care beds. ICU and hospital outcomes were available for all patients. After excluding re-admissions and patients with pre-existing chronic liver disease or cirrhosis, 723 patients with the diagnosis of acute liver failure (APACHE III-J 301.01) were admitted to ICU over the study period, accounting for $0.07 \%$ of all ICU 
admissions. This correlates to an estimated average of 3.4 admissions to intensive care with the diagnosis of ALF per million people across ANZ each year. The number of ALF admissions to ANZ ICUs increased over the ten-year period (Fig 1) at a rate that was significantly greater than population growth $(p=0.001)$.

Differences between patients with and without ALF

Differences between patients with ALF and all other ICU patients are summarised in Table 1. ALF patients were more likely to be younger, female, pregnant and immunosuppressed by disease or medical treatments. They were also less likely to have chronic severe cardiopulmonary conditions. During the first day of ICU admission, patients with ALF had higher illness acuity as measured by APACHE III and ANZROD than non-ALF ICU patients. Fewer than five per cent of ALF patients were indigenous (Australian aboriginal, Torres Strait Islander or New Zealand Maori), slightly less than the general ICU population. ALF patients were less likely to have been admitted from home and more likely to be admitted to ICU following transfer from another hospital than non-ALF patients. ALF patients exhibited hyperventilation (higher respiratory rates and lower $\mathrm{PaCO}_{2}$ ), thrombocytopaenia and developed acute renal failure more often than non-ALF patients, while limitations of treatment were similar between both groups.

Multivariable propensity analysis confirmed several key differences at the time of admission. More common amongst ALF patients were younger age, female gender, immune suppression from co-morbid disease and emergency response admissions from general wards $(p<0.0001$ for all).

\section{Patient Outcomes}

This article is protected by copyright. All rights reserved. 
A comparison of patient outcomes is outlined in Table 2. ICU and hospital mortality for ALF patients occurred in $32 \%$ and $39 \%$ respectively (similar to that predicted by the mean APACHE III (38\%) or ANZROD (34\%), whereas it occurred in only $6 \%$ and $10 \%$ of non-ALF patients ( $p<0.0001$ for both). Patients died early and mostly in the ICU. ALF was an independent predictor of mortality, with ALF patients being significantly more likely to die in hospital (OR 1.5 (1.26-1.79); $p<0.0001)$ and at 30 days (OR 1.63 (1.36-1.95); $p<0.0001)$, than patients admitted to intensive care with other diagnoses. This finding was confirmed by survival analysis (HR 1.57 (1.38-1.78); p<0.0001) (Fig 2). Independent predictors of death for ALF patients at time of admission to ICU and twenty-four hours after admission to ICU are outlined in Table 3.

Over the ten-year study period, illness severity adjusted mortality in the non-ALF ICU population decreased by $5 \%$ per year (OR $0.95(0.95-0.95) ; p=<0.0001)$; however, no significant reduction in mortality was observed for patients admitted with ALF (OR 1.01 (0.951.08); $p=0.72$ ) (Fig 3). While this temporal trend is potentially concerning, it did not meet our threshold for statistical significance.

A comparison between patients with ALF who survived versus those who died is presented in Table 4. ALF patients who survived were considerably younger and had lower rates of chronic comorbidities. Non-survivors had a higher proportion of admissions to ICU from general wards and spent longer there than those who survived. Both specific (e.g. bilirubin) and non-specific (e.g. pH, bicarbonate and platelet count) markers of liver failure were deranged to a greater extent in those ALF patients who died.

This article is protected by copyright. All rights reserved. 
Data obtained from the Australian and New Zealand Liver Transplant Registry (ANZLTR) indicate that only $8.9 \%(n=164)$ of the 1842 liver transplants during the period of our study were undertaken for acute liver failure ${ }^{37}$. Thus, it is estimated that less than $23 \%$ of ANZ ICU patients with ALF underwent ELT during the study period. Thirty-day mortality for ALF patients who were transplanted was during the study period was $12.8 \%(n=21)$, with more than $80 \%$ of these deaths occurring within the first week. Most of these deaths were due to cerebral oedema, intraoperative complications or refractory multiple organ failure. Causes of ALF in patients undergoing ELT in Australasia over the ten-year period from 2005 are outlined in Table 5.

\section{Discussion}

\section{Key findings}

In Australian and New Zealand (ANZ) data from 2005 to 2014, we found that the number of patients admitted to ICU with ALF increased at a rate exceeding population growth. Such ALF patients are younger in age, more often female, and more often require retrieval from the hospital of presentation than other ICU patients. Early during admission to ICU, they have greater illness severity and much greater mortality. While outcomes have improved for other ANZ ICU patients over time, this may be less clearly the case for patients with ALF. Finally, although the incidence, patient characteristics and overall outcomes of ALF patients admitted to ICU are similar to those reported in other regions, the ELT rate is lower than reported from other developed countries.

Relationship to previous studies

This article is protected by copyright. All rights reserved. 
Research on the characteristics and outcomes of ALF patients is overwhelmingly dominated by northern hemisphere experience (Table 6). The majority of such studies, however, are single centre and small; assess specific causes of ALF (e.g. drug induced ${ }^{22,39}$ or viral $^{40}$ ) or very specific sub-groups of ALF patients ${ }^{13,5,32}$ or children ${ }^{41-43}$, and include patients without hepatic encephalopathy ${ }^{33}$. Some high-volume transplant centres, have published large single centre observational studies $s^{7,13,29,44}$ or collaborations with similar centres ${ }^{5,18,45}$. Thus, very few studies arise from an analysis of both retrospective $e^{1,17,25,46}$ and prospective ${ }^{28}$ databases dedicated to the study of severe hepatic failure. Moreover, even when they do originate from the largest and most inclusive studies, such as The Acute Liver Failure Study Group (ALFSG), they cannot evaluate an entire population (the ALFSG enrolled only about $6.5 \%$ of potential ALF cases across the United States) and derive their data only from select specialist liver centres ${ }^{28}$. This approach creates a major selection bias and likely markedly influences ELT rates. In addition, over forty different definitions of ALF have been described ${ }^{47}$, limiting the potential comparative and external validity of such studies. Of further relevance to our study, recent data are scarce, with very few studies covering the period up to 2014 . In addition, only five previous studies evaluated a larger number of ALF patients than assessed in our study; however, these were also either single-centre, included only a very small proportion of the overall number of ALF patients in the relevant region, or were heavily skewed to specialist liver transplantation hospitals. In contrast to the above studies, our investigation is highly inclusive, recent, and captures almost all acute liver failure admissions to ICU in ANZ. As such, our findings are less affected by the over representation of highly specialised liver centres and are the first to provide population data for two countries. Finally, in several previous studies including high-activity transplantation centres, the approach to reporting overall survival, transplant-free survival and ELT survival varied considerably so that 
direct comparisons are challenging. Nonetheless, the number of ALF patients receiving ELT in most studies has consistently been approximately $50 \%$ or more $\mathrm{e}^{14,15,18,23,29}$. In this regard, the ELT rate in ANZ is amongst the lowest reported in the literature from developed countries where mature cadaveric organ donation programs exist. Knowledge of the definition and dominant causes of ALF for each region would be required to best interpret and validly compare ELT utilisation rates, however. Given the possibly limited role for ELT in paracetamol induced $\mathrm{ALF}^{48}$ and the potentially high rates of this cause of ALF in Australia $^{31,49,50}$, the low overall use of ELT in ANZ may reflect such differences in the aetiology of ALF.

Despite the above limitations, women made up 60\% of ALF patients in ANZ, a finding that is strikingly similar to previous reports (Table 6). Hospital mortality for ALF in ANZ was 39\%, also very similar to studies. The average age of patients with ALF in our study was nearly 50 years, which is older than that reported in any previous study. Given that older age is an independent predictor of poor outcome, the mortality rate we observed in ANZ may compare favourably with those from previous reports.

\section{Implications}

Our findings imply that the population incidence of ALF patients treated in ICU is increasing in ANZ. While data on aetiology was not available, other important characteristics of ALF patients in ANZ are similar to those reported in other developed countries, except for the older age of ANZ patients. They also imply that mortality for these patients may not be declining over time, unlike for other critically ill ANZ patients during this period ${ }^{51}$, suggesting that disease specific interventions are important drivers of better outcomes ${ }^{52}$. Finally, they imply that ELT rate in ANZ are much lower than reported in other developed countries, but 
that despite such low rates of ELT, overall mortality is at least equivalent to that reported from other developed countries.

\section{Strengths and weaknesses}

Our study has several strengths. First, the data were derived from one of the largest, most inclusive and comprehensive intensive care databases in the world, with very high levels of data completeness and reliable recording of patient outcomes ${ }^{53}$. The findings come from over one million ICU patient episodes and describe patient characteristics at the time of ICU admission, illness severity and hospital outcomes across the entire population of two countries for the whole of a ten-year period. Through the exclusion of patients coded for preexisting liver disease, we can be confident of ALF classification. Second, through the collection of 109 clinical variables on every patient, we could define illness severity and patient outcomes in detail. This allows comparisons between patient groups across a range of parameters and provides insights on clinically relevant differences. Third, the data collected for submission to the CORE APD includes APACHE III variables (including age, acute physiology, admission diagnosis and chronic health status) that are widely collected by ICU clinicians around the world. This provides an opportunity for future comparisons with studies from other jurisdictions where APACHE III data are routinely collected as part of existing practice. Finally, data obtained from the ANZ Liver Transplant Registry, which records all liver transplant related activity in ANZ, including all instances of liver transplantation for ALF allowed us to derive an approximate population-based ELT rate for both countries.

Our study has several potential weaknesses. As with all retrospective research based on administrative processes for data collection, there is the possibility of miscoding or other 
similar errors. The incidence of ALF might be underestimated if the reason for admission was incorrectly recorded as overdose, coma or severe sepsis. However, as the data are used for comparative benchmarking purposes, units that submit data ensure that processes are accurate and reliable and data quality is regularly evaluated for the purposes of quality improvement. This system also has data quality processes to evaluate for systemic errors and provide regular reporting to all submitting units regarding errors. Another weakness is that the database records only the admission diagnosis (acute liver failure in this case), but not the underlying cause. As such, we are unable to determine the aetiology of ALF in our cohort, preventing exploration of the relationship between aetiology and outcomes, and limiting the comparison with other reports. A further issue is that while most ALF patients are critically ill and require ICU admission, it is possible that a small number are managed on general wards and therefore not captured by the CORE APD. While we evaluated over one million ICU admissions, the actual number of patients with ALF was very small, limiting our ability to draw firm conclusions about some comparisons, such as changes in outcome over time, which failed to reach our criteria for statistical significance. Finally, we could only analyse data about illness and therapies as collected for the purposes of routine submission to the database which means that potentially relevant information (lactate, INR, liver function tests) was not available for analysis. Several previous studies have demonstrated that outcomes for ALF are highly dependent on these factors ${ }^{5,26,54,55}$, and a more detailed analysis that is inclusive of these additional considerations may assist in predicting outcomes and the advancement of treatment strategies.

\section{Conclusion}

This article is protected by copyright. All rights reserved. 
In conclusion, the current study is one the largest and, to our knowledge, the most inclusive study of ALF to date. Our findings confirm that ALF patients are a unique population amongst the critically ill, with a high mortality despite relatively young age. Gender patterns and patient characteristics are consistent with previous studies, but ANZ patients are older and, in keeping with our hypothesis, less likely to undergo ELT than patients in other regions. However, their overall outcomes are similar to those previously reported in the literature. These findings provide the basis for future interventional trials of ALF management among such patients in ANZ.

Acknowledgments:

Thanks to Glenda Balderstone of the ANZLTR for assistance with access to transplantation related data. 


\section{References}

1. Bower WA, Johns M, Margolis HS, Williams IT, Bell BP. Population-based surveillance for acute liver failure. Am J Gastroenterol 2007;102:2459-63.

2. O'Grady JG, Schalm SW, Williams R. Acute liver failure: redefining the syndromes. Lancet 1993;342:273-5.

3. Bernal W, Wendon J. Acute liver failure. N Engl J Med 2013;369:2525-34.

4. Lee WM, Squires RH, Jr., Nyberg SL, Doo E, Hoofnagle JH. Acute liver failure: Summary of a workshop. Hepatology 2008;47:1401-15.

5. Brandsaeter B, Hockerstedt K, Friman S, et al. Fulminant hepatic failure: outcome after listing for highly urgent liver transplantation-12 years experience in the nordic countries. Liver Transpl 2002;8:1055-62.

6. Gotthardt D, Riediger $\mathrm{C}$, Weiss $\mathrm{KH}$, et al. Fulminant hepatic failure: etiology and indications for liver transplantation. Nephrol Dial Transplant 2007;22 Suppl 8:viii5-viii8.

7. Marudanayagam R, Shanmugam V, Gunson B, et al. Aetiology and outcome of acute liver failure. HPB : the official journal of the International Hepato Pancreato Biliary Association 2009;11:429-34.

8. Acharya SK, Batra Y, Hazari S, Choudhury V, Panda SK, Dattagupta S. Etiopathogenesis of acute hepatic failure: Eastern versus Western countries. Journal of Gastroenterology and Hepatology 2002;17:S268-S73.

9. Acharya SK, Dasarathy S, Kumer TL, et al. Fulminant hepatitis in a tropical population: clinical course, cause, and early predictors of outcome. Hepatology 1996;23:1448-55.

10. Acharya SK, Panda SK, Saxena A, Gupta SD. Acute hepatic failure in India: a perspective from the East. J Gastroenterol Hepatol 2000;15:473-9.

11. Adukauskiene D, Dockiene I, Naginiene R, Kevelaitis E, Pundzius J, Kupcinskas L. Acute liver failure in Lithuania. Medicina (Kaunas, Lithuania) 2008;44:536-40.

12. Areia M, Romaozinho JM, Ferreira M, Amaro P, Leitao MC. Fulminant hepatic failure: a Portuguese experience. Eur J Gastroenterol Hepatol 2007;19:665-9.

13. Bhatia V, Singhal A, Panda SK, Acharya SK. A 20-year single-center experience with acute liver failure during pregnancy: is the prognosis really worse? Hepatology 2008;48:1577-85.

14. Escorsell À, Mas A, de la Mata M. Acute liver failure in Spain: Analysis of 267 cases. Liver Transplantation 2007;13:1389-95.

15. Fabrega E, Mieses MA, Tern A, et al. Etiologies and Outcomes of Acute Liver Failure in a Spanish Community. International Journal of Hepatology 2013;2013:5.

16. Forde KA, Reddy KR, Troxel AB, Sanders CM, Lee WM, Acute Liver Failure Study G. Racial and ethnic differences in presentation, etiology, and outcomes of acute liver failure in the United States. Clinical gastroenterology and hepatology : the official clinical practice journal of the American Gastroenterological Association 2009;7:11216.

17. Fujiwara K, Mochida S, Matsui A, et al. Fulminant hepatitis and late onset hepatic failure in Japan. Hepatology research : the official journal of the Japan Society of Hepatology 2008;38:646-57.

18. Hadem J, Tacke F, Bruns T, et al. Etiologies and outcomes of acute liver failure in Germany. Clinical gastroenterology and hepatology : the official clinical practice journal of the American Gastroenterological Association 2012;10:664-9 e2. 
19. Hiramatsu A, Takahashi S, Aikata $\mathrm{H}$, et al. Etiology and outcome of acute liver failure: retrospective analysis of 50 patients treated at a single center. J Gastroenterol Hepatol 2008;23:1216-22.

20. Khuroo MS. Acute liver failure in India. Hepatology 1997;26:244-6.

21. Khuroo MS, Kamili S. Aetiology and prognostic factors in acute liver failure in India. J Viral Hepat 2003;10:224-31.

22. Larson AM, Polson J, Fontana RJ, et al. Acetaminophen-induced acute liver failure: results of a United States multicenter, prospective study. Hepatology 2005;42:136472.

23. Shakil AO, Kramer D, Mazariegos GV, Fung JJ, Rakela J. Acute liver failure: clinical features, outcome analysis, and applicability of prognostic criteria. Liver Transpl 2000;6:163-9.

24. Shalimar, Subrat KA, William ML. Worldwide differences in acute liver failure. Critical Care in Acute Liver Failure: Future Medicine Ltd; 2013:32-46.

25. Sugawara K, Nakayama N, Mochida S. Acute liver failure in Japan: definition, classification, and prediction of the outcome. Journal of gastroenterology 2012;47:849-61.

26. Canbay A, Jochum C, Bechmann LP, et al. Acute liver failure in a metropolitan area in Germany: a retrospective study (2002 - 2008). Z Gastroenterol 2009;47:807-13.

27. Fujiwara K, Yasui S, Yonemitsu Y, et al. Fixed point observation of etiology of acute liver failure according to the novel Japanese diagnostic criteria. Journal of hepatobiliary-pancreatic sciences 2015;22:225-9.

28. Reuben A, Tillman H, Fontana RJ, et al. Outcomes in Adults With Acute Liver Failure Between 1998 and 2013: An Observational Cohort Study. Ann Intern Med 2016;164:724-32.

29. Bernal W, Hyyrylainen A, Gera A, et al. Lessons from look-back in acute liver failure? A single centre experience of 3300 patients. J Hepatol 2013;59:74-80.

30. Canbay A, Tacke F, Hadem J, Trautwein C, Gerken G, Manns MP. Acute liver failure: a life-threatening disease. Dtsch Arztebl Int 2011;108:714-20.

31. Gow PJ, Jones RM, Dobson JL, Angus PW. Etiology and outcome of fulminant hepatic failure managed at an Australian liver transplant unit. J Gastroenterol Hepatol 2004;19:154-9.

32. Reddy KR, Ellerbe C, Schilsky M, et al. Determinants of outcome among patients with acute liver failure listed for liver transplantation in the United States. Liver Transpl 2016;22:505-15.

33. Koch DG, Speiser JL, Durkalski V, et al. The Natural History of Severe Acute Liver Injury. Am J Gastroenterol 2017;112:1389-96.

34. Bernal W, Lee WM, Wendon J, Larsen FS, Williams R. Acute liver failure: A curable disease by 2024? J Hepatol 2015;62:S112-20.

35. Kasza J, Moran JL, Solomon PJ, Outcome AN-ANZICSCf, Resource Evaluation C. Evaluating the performance of Australian and New Zealand intensive care units in 2009 and 2010. Stat Med 2013;32:3720-36.

36. Paul E, Bailey M, Pilcher D. Risk prediction of hospital mortality for adult patients admitted to Australian and New Zealand intensive care units: development and validation of the Australian and New Zealand Risk of Death model. Journal of critical care 2013;28:935-41.

37. Australia \& New Zealand Liver Transplant Registry 26th Report. Brisbane, Queensland, Australia: Australia and New Zealand Liver Transplant Registry; 2015. 
38. von Elm E, Altman DG, Egger $M$, et al. The Strengthening the Reporting of Observational Studies in Epidemiology (STROBE) statement: guidelines for reporting observational studies. Lancet 2007;370:1453-7.

39. Reuben A, Koch DG, Lee WM, Acute Liver Failure Study G. Drug-induced acute liver failure: results of a U.S. multicenter, prospective study. Hepatology 2010;52:2065-76.

40. Taylor RM, Davern T, Munoz S, et al. Fulminant hepatitis A virus infection in the United States: Incidence, prognosis, and outcomes. Hepatology 2006;44:1589-97.

41. Kortsalioudaki C, Dassios T, Bansal S, et al. OUTCOME OF IDIOPATHIC ACUTE LIVER FAILURE IN CHILDREN: 15 YEARS, SINGLE CENTRE EXPERIENCE: PH220. J Pediatr Gastroenterol Nutr 2005;40:684-5.

42. Squires RH, Jr., Shneider BL, Bucuvalas J, et al. Acute liver failure in children: the first 348 patients in the pediatric acute liver failure study group. J Pediatr 2006;148:652-8.

43. Durand $P$, Debray $D$, Mandel $R$, et al. Acute liver failure in infancy: a 14-year experience of a pediatric liver transplantation center. J Pediatr 2001;139:871-6.

44. Faria L, Ichai P, Saliba F, Azoulay D, Antonini T, Ben Hamida S. Etiology, outcome and early cause of death in 500 patients with acute liver failure: 20 year single centre experience. Hepatology 2006;44:370A.

45. Escorsell A, Mas A, de la Mata M. Acute liver failure in Spain: analysis of 267 cases. Liver Transpl 2007;13:1389-95.

46. Wei $G$, Bergquist $A$, Broome $U$, et al. Acute liver failure in Sweden: etiology and outcome. Journal of internal medicine 2007;262:393-401.

47. Wlodzimirow KA, Eslami S, Abu-Hanna A, Nieuwoudt M, Chamuleau RA. Systematic review: acute liver failure - one disease, more than 40 definitions. Aliment Pharmacol Ther 2012;35:1245-56.

48. Gow PJ, Warrillow S, Lontos S, et al. Time to review the selection criteria for transplantation in paracetamol-induced fulminant hepatic failure? Liver transplantation : official publication of the American Association for the Study of Liver Diseases and the International Liver Transplantation Society 2007;13:1762-3.

49. Gow PJ, Angus PW, Smallwood RA. Transplantation in patients with paracetamolinduced fulminant hepatic failure. Lancet 1997;349:651-2.

50. Gow PJ, Smallwood RA, Angus PW. Paracetamol overdose in a liver transplantation centre: an 8-year experience. J Gastroenterol Hepatol 1999;14:817-21.

51. Kaukonen KM, Bailey M, Suzuki S, Pilcher D, Bellomo R. Mortality related to severe sepsis and septic shock among critically ill patients in Australia and New Zealand, 2000-2012. Jama 2014;311:1308-16.

52. Zimmerman JE, Kramer AA, Knaus WA. Changes in hospital mortality for United States intensive care unit admissions from 1988 to 2012. Crit Care 2013;17:R81.

53. ANZICS. ANZICS Centre for Outcome and Resource Evaluation Annual Report 20132014. Melbourne, Australia: ANZICS; 2015 September 2015.

54. Brems JJ, Hiatt JR, Ramming KP, Quinones-Baldrich WJ, Busuttil RW. Fulminant hepatic failure: the role of liver transplantation as primary therapy. Am $\mathrm{J}$ Surg 1987;154:137-41.

55. Castaldo ET, Chari RS. Liver transplantation for acute hepatic failure. HPB : the official journal of the International Hepato Pancreato Biliary Association 2006;8:29-34.

56. Yantorno SE, Kremers WK, Ruf AE, Trentadue JJ, Podesta LG, Villamil FG. MELD is superior to King's college and Clichy's criteria to assess prognosis in fulminant hepatic failure. Liver Transpl 2007;13:822-8. 


\section{University Library}

\section{- M M N E R VA A gateway to Melbourne's research publications}

Minerva Access is the Institutional Repository of The University of Melbourne

Author/s:

Warrillow, S;Bailey, M;Pilcher, D;Kazemi, A;McArthur, C;Young, P;Bellomo, R

Title:

Characteristics and outcomes of patients with acute liver failure admitted to Australian and New Zealand intensive care units

Date:

2019-07-01

Citation:

Warrillow, S., Bailey, M., Pilcher, D., Kazemi, A., McArthur, C., Young, P. \& Bellomo, R. (2019). Characteristics and outcomes of patients with acute liver failure admitted to Australian and New Zealand intensive care units. INTERNAL MEDICINE JOURNAL, 49 (7), pp.874-885. https://doi.org/10.1111/imj. 14167.

Persistent Link:

http://hdl.handle.net/11343/286107 